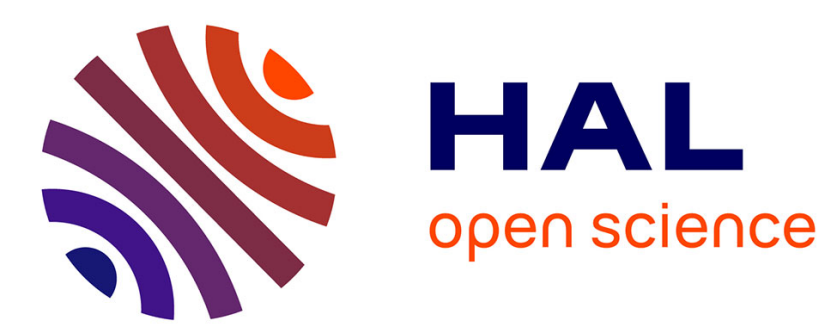

\title{
Deux ou trois choses nouvelles en géographie urbaine
}

\author{
Denise Pumain, Thérèse Saint-Julien
}

\section{To cite this version:}

Denise Pumain, Thérèse Saint-Julien. Deux ou trois choses nouvelles en géographie urbaine. EspacesTemps.net, 1984, 26-27-28, pp.51-61. 10.3406/espat.1984.3217 . halshs-01520394

\section{HAL Id: halshs-01520394 https://shs.hal.science/halshs-01520394}

Submitted on 10 May 2017

HAL is a multi-disciplinary open access archive for the deposit and dissemination of scientific research documents, whether they are published or not. The documents may come from teaching and research institutions in France or abroad, or from public or private research centers.
L'archive ouverte pluridisciplinaire HAL, est destinée au dépôt et à la diffusion de documents scientifiques de niveau recherche, publiés ou non, émanant des établissements d'enseignement et de recherche français ou étrangers, des laboratoires publics ou privés. 


\section{Deux ou trois choses nouvelles en géographie urbaine}

\section{Denise Pumain, Thérèse Saint-Julien}

\section{Résumé}

Au delà de la diversité présente et de l'ampleur des évolutions qui marquent, dans les hiérarchies de taille et de fonctions, les systèmes de villes, peut-on proposer des régularités suffisamment fortes pour apparaître comme des invariants ? Denise Pumain et Thérèse Saint-Julien font le point sur une question, familière pour elles et décisive pour l'approche sociale de l'espace.

\section{Citer ce document / Cite this document :}

Pumain Denise, Saint-Julien Thérèse. Deux ou trois choses nouvelles en géographie urbaine. In: Espaces Temps, 26-28, 1984. L'espace en société. Géographies d'aujourd'hui. pp. 51-61;

doi : 10.3406/espat.1984.3217

http://www.persee.fr/doc/espat_0339-3267_1984_num_26_1_3217

Document généré le 21/03/2016 


\title{
Deux ou trois choses nouvelles en géographie urbaine.
}

\author{
Denise Pumain et Therèse Saint-Julien
}

\begin{abstract}
Au DELÀ DE LA DIVERSITÉ PRÉSENTE ET DE L'AMPLEUR DES ÉVOLUTIONS QUI MARQUENT, DANS LES HIÉRARCHIES DE TAILLE ET DE FONCTIONS, LES SYSTEMMES DE VILLES, P.EUT-ON PROPOSER DES REGULARITÉS SUFFISAMMENT FORTES POUR APPARAITTRE COMME DES INVARIANTS? DENISE Pumain ET THÉRÉSE SAINT-JUL IEN FONT LE POINT SUR UNE QUESTION, FAMILIĖRE POUR ELLES ET DÉCISSIVE POUR L'APPROCHE SOCIALE DE L'ESPACE .
\end{abstract}

... Je pourrais te dire de combien de marches sont faites les rues'en escalier, de quelle forme sont les arcs des portiques, de quelles feuilles de zinc les toits sont recouverts; mais déjà je sais que ce serait ne rien te dire. Ce n'est pas de cela qu'est faite la ville, mais des relations entre les mesures de son espace et les événements de son passé ...

Italo Calvino, Les villes invisibles, Paris, Seuil, 1974, p.15.

Il est incontestable que la géographie a profondément changé depuis une quinzaine d'années. Ce serait ne donner qu'une interprétation superficielle de ce changement de s'en tenir à ses marques les plus visibles: introduction de techniques statistiques, de la modélisation, usage de l'informatique et des images produites par satellite, ouverture à d'autres sciences, incorporation de nouveaux thèmes: le social, les comportements, les représentations. Dans cette courte note, nous voudrions insister davantage sur le changement intervenu dans la manière de concevoir le questionnement géographique, d'interpréter la contribution de la géographie à la connaissance des sociétés. Ce changement-là résulte de l'apport d'attitudes scientifiques différentes, de l'assimilation de nouveaux paradigmes qui étaient "dans l'air", et aussi de la réflexion sur les résultats des études empiriques et expérimentales permises par les techniques nouvelles. Il n'est certes pas question que nous rendions compte ici de ces changements pour l'ensemble de la discipline, ni même pour la. totalité de la géographie urbaine française. L'on nous pardonnera de nous limiter à quelques domaines et à un type d'approches particulier. 


\section{Des invariants en géographie urbaine.}

Les vieux démons de l'indéterminisme qui, en géographie, ont pendant quelques décennies condamné l'approche nomothétique et orienté la recherche vers la seule production monographique se sont un peu assoupis. Parallèlement certains géographes ont été sensibles au fait que les déterminismes sociaux ne sont pas, plus que d'autres, en mesure de fonder à eux seuls correctement la géographie comme science. En effet leur attention a été attirée par le fait que des régularités, des répétitions apparaissent dans l'organisation des trames, des flux, des territoires à la surface de la terre. Ils se sont donc attachés à définir la nature, la forme et l'ampleur de ces régularités spatiales ou invariants géographiques et, partant de cette connaissance, ils ont cherché une formulation des règles, des lois, des processus susceptibles de rendre compte de leur genèse ou de leur conservation.

Une telle démarche cherche toujours à répondre à la question fondamentale: pourquoi cela est-il là et pas ailleurs?, mais elle privilégie la démarche comparative, substitut en sciences sociales de la démarche expérimentale, et dans une première étape, la recherche des invariants spatiaux. La notion d'invariance en géographie n'est bien entendu que relative, elle $n$ 'en est pas moins essentielle. En effet, on ne peut trouver à la surface de la terre que des conditions relativement proches d'apparition d'un même phénomène. Donc il n'y a jamais que des probabilités plus ou moins fortes d'observer certaines configurations dans un ensemble de conditions donné. Ces réserves ne remettent pas en cause la pertinence de la recherche d'invariants spatiaux; elles en précisent simplement le cadre, la géographie n'échappant pas au lot commun de l'ensemble des sciences sociales en matière de définition d'invariance.

La connaissance de i'urbain a particulièrement bénéficié des apports de ces nouvelles perspectives de recherche. L'existence de régularités a été démontrée à différents niveaux géographiques.

\subsection{A propos des dimensions latentes d'un système urbain national.}

Dans un souci d'inventaire de la diversité des physionomies urbaines, les géographes se sont attachés à caractériser chaque cas observé et à dresser des typologies de villes. Ils ont, dans cet inventaire, privilégié la caractérisation de l'activité des villes (ville de marché, ville militaire, ville portuaire, ville de pèlerinage, etc...). Répertoire ordonné des situations observées, l'établissement de ces typologies est resté pendant longtemps une fin en soi. En témoignent encore aujourd'hui en France, certains manuels de géographie urbaine. Nous pensons que dans une première étape, ce travail d'inventaire est indispensable au développement de toute discipline. En cela la géographie urbaine $n^{\prime}$ a fait que se plier à une exigence qu'ont, dans un premier temps, satisfaite toutes les sciences humaines ou naturelles. Elle s'en est, peut-être plus que d'autres, pendant très ou trop longtemps satisfaite.

Plusieurs constatations ont permis un dépassement de la démarche strictement typologique. On a d'une part observé que les types recensés dans un territoire donné se retrouvaient, toutes choses égales par ailleurs, sur d'autres territoires, et parfois dans des configurations géographiques à bien des égards comparables. On a d'autre part disposé d'un nombre croissant d'indices témoignant de l'interdépendance des villes à l'intérieur d'un même espace national. Des recherches ont alors été menées pour identifier les principes qui fondent la diversité des 
situations urbaines à l'intérieur d'un même espace national, et pour évaluer le degré de leur invariance géographique. Quels enseignements peut-on aujourd'hui tirer de ces travaux?

Partant d'analyses systématiques effectuées dans un très grand nombre de pays, sur des ensembles nombreux de villes décrites par une très grande variété de caractères socio-économiques et démographiques, des résultats convergents ont parmis d'identifier les dimensions latentes*, suivant lesquelles se structure un système urbain national et par rapport auxquelles peut se définir la position relative de chacune de ces villes dans l'ensemble. Ces dimensions aujourd'hui connues sont nombreuses. Nous en évoquons ici deux dont l'universalité est désormais quasiment démontrée.

\subsubsection{Hiérarchie des tailles.}

La hiérarchie des tailles des unités urbaines, associée à une hiérarchie de la distribution interurbaine du pouvoir économique et des diverses expressions des fonctions de desserte et d'encadrement du territoire, et souvent combinée à certaines caractéristiques de la société urbaine, est un des principes majeurs de la différenciation interurbaine, et peut-être le plus important révélateur de l'organisation du système urbain.

Les vérifications et approfondissements théoriques qui ont pu être faits, à partir et autour de la loi rang-taille d'une part et de la théorie des places centrales d'autre part, ont été une autre manière de démontrer l'universalité de ce principe et sa force de structuration dans un réseau urbain. La loi rang-taille a depuis longtemps décrit le fait que, pour un territoire donné, il existe toujours un petit nombre de grandes villes et un grand nombre de petites villes et que la diminution du nombre des villes suit une progression géométrique à peu près régulière, lorsqu'on considère des catégories de taille de plus en plus élevée. Des vérifications systématiques et approfondies ont permis aujourd'hui d'affiner la formulation de cette loi (Béguin, 1979, Pumain, 1982). La théorie des places centrales, attachée au nom de Christaller (1933), représente la première tentative importante pour expliquer et modéliser le nombre, la taille et l'espacement des villes, en fonction de l'importance de la population qu'elles desservent. Nous ne rappellerons pas ici les bases désormais classiques de cette théorie. L'apport récent est que la portée explicative de cette théorie a pu être vérifiée dans des contextes géographiques très différents, mettant en évidence la généralité de certains des fondements donnés à la distribution spatiale d'un réseau de villes dans des territoires très divers (ainsi pour la France Hautreux, Lecourt, Rochefort, 1963; pour les Etats-Unis, Berry, 1967; pour la Chine, Skinner, 1964). Lès vérifications effectuées ont aussi permis un approfondissement théorique, soit pour améliorer les outils d'analyse (Cauvin, Reynaud, 1980), soit pour réexplorer les fondements cognitifs et comportementaux de la centralité (Cauvin, 1984).

\subsubsection{Base économique.}

La base économique des villes est à l'origine d'un deuxième princi-

* Les techniques utilisées permettent de spécifier très précisément le degré d'indépendance ou de dépendance (corrélation) et de hiérarchiser l'importance de ces dimensions (Klein, Pruvot, 1984 ). 
pe de différenciation des situations urbaines. Il est l'expression de la diversité des spécialisations fonctionnelles des centres. Il traduit donc les arrangements spatiaux de l'activité économique qui résultent de la distribution géographique des facteurs de production et des rapports qui s'établissent, à un moment donné, entre leur localisation et celle de l'activité. Il reflète aussi le degré d'inertie que manifestent par la suite les activités à l'égard des localisations urbaines. Cette composante de la structure d'un réseau urbain est une des expressions fortes des interactions économiques qu'entretiennent entre elles les villes. Elle contribue donc à définir la position de chacune dans l'ensemble d'une manière qui ne recouvre pas les inégalités interurbaines définies par la dimension hiérarchique.

Cette indépendance entre la base économique des villes et leur taille a déjà été signalée pour d'autres pays que la France (Berry, 1972). En revanche, l'association étroite de la dimension économique à la distribution sociale, voire démographique et cognitive des villes qui a été identifiée sous le nom générique d'"image de marque" (Pumain, Saint-Julien, 1978), n'a jusqu'ici été observée que pour le système urbain français, mais rien n'indique qu'elle ne puisse être mise en évidence dans d'autres pays.

\subsection{A propos des régularités de la configuration interne des villes.}

La préoccupation $n^{\prime}$ 'est pas nouvelle. Dès le $19^{\circ}$ siècle (Robic, 1982), des textes rappelaient que la structuration des espaces intra-urbains obéit à un certain nombre de règles, que les notions de distance, de rente foncière et de ségrégation sociale sont en mesure d'éclairer. Il a fallu attendre les travaux qui se sont développés dans les années vingt autour de l'école de sociologie urbaine de Chicago pour qu'une plus grande attention soit portée à la définition des principes qui structurent l'espace des villes (Grafmeyer, 1979). Dans la géographie urbaine française, ces perspectives ont été longtemps occultées par la priorité données aux études de morphologie urbaine, qui se sont plus attachées à dégager les spécificités physionomiques des villes, qu'à rechercher les principes de leur organisation territoriale.

Quelques-uns des principes qui structurent l'espace résidentiel des villes sont maintenant bien établis. Confrontés à des situations urbaines très diverses, villes nord-américaines: Chicago (Berry, Horton, 1970), Montréal (Racine, 1973), Québec, Edmonton (Bailly, Polese, 1976, 1978), par exemple; villes françaises: Nîmes, Montpellier, Perpignan (Vigouroux, Volle, 1982), Strasbourg (Klein, Pruvot, 1984), Rouen (Guermond, 1981), Paris (Noin, Chauviré, 1980), ou indienne: Calcutta (Bose, 1968), ces principes ont fait preuve d'une réelle robustesse.

Le premier d'entre eux, dit "facteur socio-économique", décrit les ségrégations qui, dans les villes, sont dues aux disparités des situations socio-économiques des populations. Il représente la composante de la structure résidentielle des agglomérations qui fonde les plus grandes disparités entre les aires de voisinage. Il résulte de la recherche par les populations urbaines d'externalités économiques et sociales. Le deuxième met bien en évidence le fait qu'à un moment donné, la configuration géographique des aires résidentielles est indissociable de la structuration par âge des populations. En d'autres termes, on a pu montrer qu'il existe une relation assez générale entre la position de ces populations dans le cycle de vie et leur localisation dans l'espace urbain. Un troisième principe a également été mis en évidence. Son poids 
dans l'organisation des espaces résidentiels est un peu moins grand que celui des précédents, mais sa généralité mérite qu'on s'y arrête. Il s'agit d'une composante qui décrit les disparités ethniques de la population d'une ville et les ségrégations résidentielles qui y correspondent.

On a pu dans les villes nord-américaines associer à chacun des principes de la structuration de l'espace résidentiel des villes une configuration spatiale particulière (schéma sectoriel pour le premier, concentrique pour le deuxième, etc...). Quand ces principes se sont avérés déterminants pour expliquer l'organisation résidentielle des unités urbaines dans des aires culturelles plus ou moins marquées par un très ou plus ancien processus d'urbanisation, il va de soi que l'on a dû faire intervenir le processus de structuration historique de la ville, pour définir les configurations géographiques qui leur correspondent aujourd'hui.

Les distributions des activités économiques imposent aussi à l'espace urbanisé certaines règles d'organisation dont la plus importante est que les activités tertiaires tendent à se localiser dans la ville suivant les principes de la centralité, formulés initialement à une tout autre échelle (espaces régionaux ou nationaux) par la théorie des places centrales. Les récents travaux nous persuadent de la validité de ce transfert théorique de l'échelle d'un système de villes à celle d'un élément de ce système (Vance, 1962, Berry, Horton, 1970, Racine, 1973, par exemple).

L'évocation de ce transfert pose aujourd'hui une réelle question que nous aurons vraisemblablement intérêt à débroussailler dans les prochaines années. Tout dans les observations qui précèdent porte à penser que la configuration d'ensemble d'un système de villes et la structure interne de chacun de ses éléments sont en constante interaction. On peut raisonnablement faire l'hypothèse que des liens existent entre les invariants du système, dont nous venons de rappeler quelques traits essentiels, et les règles (pour une part sans doute encore mal connues) suivant lesquelles semblent s'organiser les espaces intra-urbains. L'existence de ces liens est encore aujourd'hui mal démontrée alors que, si l'on aborde le problème en termes dynamiques, considérant croissance et devenir des villes dans la dynamique du système, des liaisons commencent à apparaître. Il y a donc là un encouragement à mener des recherches dans ce sens.

\section{De la dynamique des systèmes urbains.}

Les innovations les plus prometteuses et à terme les plus satisfaisantes de la recherche urbaine sont sans doute celles qui ont trait à l'évolution des systèmes urbains. Réintégrant le temps dans l'analyse, elles permettent d'approcher de plus près le fonctionnement de ces systèmes et de mettre à l'épreuve les hypothèses qui ont été formulées pour en expliquer le fondement.

\subsection{Croissance urbaine et taille des villes.}

Ainsi, les études de la croissance urbaine qui étaient menées dans les années soixante étaient des études monographiques qui s'attachaient à découvrif dans la situation de la ville, son site, son histoire passée, le dynamisme de ses édiles..., le pourquoi de son explosion démographique 
et spatiale ou de sa stagnation. Les comparaisons faites sur plusieurs intervalles successifs intercensitaires tentaient d'interpréter les fluctuations de cette évolution dans les mêmes termes. Or toutes ces études oubliaient une composante importante de la croissance urbaine, qui relève d'un autre échelon géographique d'interprétation, et probablement aussi d'une autre échelle temporelle, et qui correspond à la logique d'un système des villes.

Qu'a montré en effet une étude sur très longue durée (depuis le début du $19^{\circ}$ siècle) de l'évolution de la trame urbaine française? Elle a montré la très grande permanence de la forme statistique de la distribution des villes, la relative stabilité des positions des plus grandes villes dans la hiérarchie (quasi stabilité de la primatie parisienne, c'est-à-dire du rapport entre la taille de la capitale et celle des agglomérations de second rang; parmi les villes qui sont aujourd'hui les plus grandes, une importante proportion occupait déjà un rang élevé dans la hiérarchie urbaine au début du $19^{\circ}$ siècle, voire longtemps auparavant). Un certain système de peuplement s'est donc maintenu avec des caractéristiques déterminées à travers près de deux siècles (sinon plus), à travers la première et la deuxième révolution industrielle et l'exode rural, pendant la stagnation d'entre deux guerres comme pendant le boom démographique et économique et la phase d'urbanisation rapide des trente dernières années. La croissance démographique et les innovations économiques ont ainsi provoqué une élévation quasi continue de la taille des villes et une augmentation considérable de leur nombre (111 unités urbaines de plus de 10000 habitants en 1831,409 en 1975), sans que soient remis en cause les principaux rapports dimensionnels du système urbain français (les paramètres qui résument sa configuration par rapport à celle des autres systèmes urbains, forte permanence, grande disparité de taille, ainsi que la faible densité et la régularité du semis - cf. Pumain, 1984), ni la place relative des éléments dans le système. Surtout, il est remarquable que cette permanence résulte d'ajustements répétés aux changements, qui se produisent à partir d'un processus de répartition de la croissance urbaine qui est, lui, extrêmement fluctuant. S'il est vrai qu'en moyenne sur très longue période la croissance urbaine (quantité de population ajoutée) est proportionnelle à la taille des villes, les taux de variation (la proportion de population ajoutée) calculés sur des temps courts sont eux fondamentalement instables et imprévisibles (Henry et Guttierrez, 1977, Pumain, 1982). A chaque "instant" (sur un intervalle de quelques années) se produisent des fluctuations de la grandeur de la population des villes (tendant à faire progresser ou régresser leur position relative dans la hiérarchie) qui sont le plus souvent quasi aléatoires, et cette agitation constante a cependant pour résultat le maintien des positions hiérarchiques relatives sur le long terme. Déterminisme et aléa font ici partie du même phénomène.

Cette capacité d'auto-organisation d'un système spatial non seulelement est compatible avec, mais aussi résulte, des processus intervenant à un échelon géographique inférieur, où les finalités des éléments agissant n'ont rien à voir directement avec ce maintien de la structure du système urbain: on imagine aisément que les motivations individuelles des habitants et leurs comportements aient de tout autres déterminants. Cependant, il est probable que certaines macro-régularités issues de ces comportements individuels (dont les fortes non-linéarités seraient dues aux comportements d'imitation et d'anticipation, cf. Allen, 1981) ainsi que les contraintes opérées par le système des villes existant dans lequel ils s'insèrent, aboutissent à des régulations par un échelon géographique supérieur.

C'est un peu ce que $R$. Bussière exprime dans son dernier ouvrage 
lorsqu'il écrit: "Les habitants des villes modernes choisissent et adaptent leurs lieux de résidence de telle manière que la proportion de population qui subit un niveau donné de pression civique* reste toujours la même. Les pressions moyennes sont constantes dans le temps." (Bussière, Stovall, 1981, pp. 134-135). R. Bussière essaie de rendre compte des liaisons statistiques observées (sur un très grand nombre de villes et des périodes, dans de nombreux pays) entre la population urbaine totale et le nombre des villes d'un système urbain, et la population de chaque ville, sa densité et sa surface, à travers deux paramètres fondamentaux qui sont la tendance à l'étalement spatial (ou gradient de densité de la population à T'intérieur des villes) et la propension d'une population à se rassembler dans un nombre limité de villes. Nous avons appelé le second "principe d'agglomération" (Pumain, 1982). Il est une évaluation du degré d'inégalité, de contraste - d'asymétrie - de la distribution des tailles des villes. Dans le modèle qu'il propose, la forme exponentielle décroissante de la courbe des densités de la population d'une ville en fonction de la distance au centre, et la relation de Pareto (loi rang-taille) entre la population d'une ville et son rang dans l'ensemble des villes d'une "région" (suffisamment grande et cohérente) sont liées par une relation linéaire croissante entre taille des villes et densité. Au cours du temps, cette relation se maintient avec pour les villes de l'époque moderne une décroissance de la densité centrale et du gradient de densité (qui restent liés cependant par une relation linéaire) et une augmentation de la concentration de la distribution de la taille des villes. Tout se passe donc comme si, du fait de son appartenance au système, le devenir d'une ville était en quelque sorte contrôlé, de manière endogène et de manière exogène, avec une marge de "liberté" dont l'amplitude n'excède que rarement, en moyenne pour l'ensemble des villes, le seuil au-delà duquel la structure du système se trouverait modifiée.

\subsection{Déplacements intra-urbains et modèles entropiques.}

Une autre illustration de cette interaction entre la ville et le système de villes, qui non seulement assigne des limites au développement de chacune mais encore lui impose certaines modalités de fonctionnement, peut être donnée à propos de la géographie des déplacements intra-urbains quotidiens. A. Wilson (1970) a montré que l'on pouvait toujours comparer la distribution effective de ces déplacements à celle que l'on obtiendrait en faisant un minimum d'hypothèses indispensables: celles-ci impliquent que l'on prenne en considération la taille des populations à déplacer d'une zone à l'autre (nombre de résidents et nombre d'emplois dans chaque zone) ainsi qu'un coût total des déplacements pour l'ensemble de l'agglomération urbaine. Ces deux composantes sont nécessaires pour que le modèle calculé ait quelque ressemblance avec les déplacements que l'on observe effectivement. Or, si la première donnée (la configuration des zones d'emploi et de résidence de la ville) est bien une contrainte d'origine endogène, propre à chaque ville, on peut considérer que la seconde (le coût des déplacements) est une contrainte qui a au moins en partie une origine exogène, le coût de fonctionnement d'une agglomération ne pouvant s'établir indépendamment de ce qui se

* La "pression civique" peut être assinilée à la "pression du loyer" ou encore à la "rente foncière" associées aux localisations situées à un éloignement donné du centre. 
passe dans le reste du système de villes.

Un point nous semble à retenir dans ces démarches: les parallèles effectués entre le fonctionnement d'un ensemble de villes et celui des systèmes ouverts situés loin de l'équilibre, capables de s'auto-organiser en "structures dissipatives", n'ont pas été posés a priori - hormis dans le fait de décider d'étudier et de comparer l'évolution d'un grand nombre de villes. Mais ce sont bien les résultats d'études empiriques - au départ desquelles on déplorait d'ailleurs souvent le manque de références théoriques - qui ont conduit à proposer d'utiliser cette interprétation, qui les éclaire sans les contredire. En outre, elle ne s'appuie pas seulement sur le cas de la France, mais là encore sur des traits qui pourraient être considérés comme des invariants, du moins par rapport aux études actuellement disponibles dans d'autres pays.

\subsection{Histoire et dynamique.}

Enfin, et surtout, ces interprétations ne se situent pas hors du temps de l'histoire, mais se l'incorporent. Ainsi, $R$. Bussière signale bien que la relation linéaire entre densité de la population au centre ville et gradient de densité dans l'cnscmblc de l'agglomération ne s'est établie en France que vers 1900, au moment où se généralisait l'utilisation intra-urbaine des moyens de transport, et donc où la population acquérait un certain degré de mobilité.

De même, nous avons pu constater en observant le processus contemporain de croissance urbaine que les taux de variation de la population des villes cessaient d'être distribués de manière aléatoire d'une période sur l'autre et devenaient au contraire de plus en plus autocorrélés temporellement*, indiquant probablement une réorganisation structurelle en cours du système urbain. Une telle réorganisation a bien été mise en évidence dans l'étude des transformations qualitatives des caractères socio-économiques des villes, et interprétée comme l'émergence de nouveaux rapports centre/périphérie dans le système des villes (cf. SaintJulien, 1982, pp. 219-257).

En effet, on pcut considérer que les différenciations majeures qui apparaissent entre les villes à un moment donné, en tant que dimensions latentes du système, sont d'abord apparues progressivement, comme des fluctuations d'amplitude mineure. Même si ces fluctuations étaient d'origine extérieure au système urbain (par exemple la première ou la seconde révolution industrielle), elles ont $\mathrm{pu}$ se trouver amplifiées par le fonctionnement de ce système au point d'en transformer la structure. Ainsi, chaque fois qu'une chaîne d'innovations socio-économiques présente une distribution géographique sélective, une réorganisation de la structure du système urbain (bifurcation) est susceptible d'intervenir. Ensuite, de par le fonctionnement "normal" du système, la différenciation ainsi créée tend à se reproduire, au-delà des conditions qui l'ont fait naftre. C'est ainsi que la dimension qui rend compte aujourd'hui encore de l'essentiel des disparités socio-économiques urbaines en France est un

\footnotetext{
* Corrélation des taux de variation de la population des unités urbaines de plus de 10000 habitants: $1954-62$ et 1962-68: 0,46; 1962-68 et 1968-75: 0,57; 1968-75 et 1975-82:0,59; alors que ces corrélations sont à peu près nulles tout au long du XIXème siècle jusque vers 1880 , et de l'ordre de 0,2 ensuite.
} 
héritage de la révolution industrielle. C'est ainsi également qu'on a pu observer, en la suivant phase par phase de 1954 à 1982, l'émergence d'une nouvelle dimension latente du système urbain français. Par suite en effet des changements apparaissant d'abord comme aléatoires, puis s'amplifiant en associant plusieurs types de caractéristiques urbaines (attraction d'activités en croissance industrielle puis tertiaires et des catégories sociales à haut niveau de formation technico-administrative dans les villes des régions "centrales" - Bassin Parisien, Centre-Est - et au contraire, accumulation relative d'activités en déclin et des modes de production anciens dans des villes en situation "périphérique"), une nouvelle dimension du système urbain français est apparue. Elle a été identifiée comme celle de la "modernité" et comme la référence à l'histoire courte contemporaine (Pumain, Saint-Julien 1978, Saint-Julien 1982).

Enfin, il est à peu près certain que leṣ modalités que nous avons décrites et qui gouvernent l'évolution des systèmes de villes depuis au moins un siècle subissent des modifications telles qu'elles conduisent à des transformations qualitatives de l'organisation et du fonctionnement du système urbain. Un paramètre essentiel est en effet en train de changer d'ordre de grandeur: il s'agit bien sûr de la vitesse de circulation de l'information, qui est susceptible d'affecter la relation des individus à la distance physique et les formes de leur mobilité, donc la configuration du système de peuplement à l'intérieur du territoire.

Le fait nouveau pour la géographie urbaine, qui dérive de son ouverture à l'interprétation systémique et à la théorie des bifurcations, est que ces théories s'accompagent désormais d'outils mathématiques de modélisation et de simulation des transformations qualitatives des systèmes. Il y a en effet bien longtemps que l'on a reconnu l'existence d'un "système général des villes" ( $\mathrm{J}$. Reynaud en 1841, cité par M.-C. Robic, 1982) ou relevé des discontinuités en géographie (Brunet, 1967). Mais c'est seulement depuis quelques années que l'on sait reproduire par simulation des histoires urbaines - au moyen de modèles dynamiques non linéaires. P. Allen, de l'école de Bruxelles (Allen et al., 1981), s'appuyant sur la théorie des bifurcations, A. Wilson utilisant la théorie des catastrophes (Wilson, 1982), ont pu reproduire, sur des exemples de villes fictifs, des transformations qualitatives de leur configuration spatiale. Des applications à des villes réelles sont en cours et devraient permettre de tester la généralité - l'invariant - des processus dynamiques intégrés dans ces modèles. De telles expérimentations permettent ainsi de mettre à l'épreuve des hypothèses théoriques formulées au regard des observations empiriques et d'en mesurer l'utilité.

D'ici quelque temps, ces modèles nous permettront-ils de ne plus seulement expérimenter rétrospectivement, mais, à partir de la connaissance acquise sur le fonctionnement passé, de prévoir l'évolution des villes? Le principe même de la théorie exclut toute prévision certaine, mais permet d'associer à tel ou tel état vers lequel peut évoluer le système une certaine probabilité. Par ailleurs, aucun de ces modèles ne prétend prendre en compte la totalité des variables urbaines, seulement celles qui semblent déterminantes par rapport à un problème donné. L'avenir de la recherche urbaine est donc encore largement ouvert!...

Pour résumer un peu l'esprit des quelques approches nouvelles dont nous avons voulu faire état pour la géographie urbaine, il nous semble qu'on pourrait formuler les observations suivantes: au lieu de rechercher l'explication des distributions observées par une causalité externe, qu'elle relève d'un déterminisme physique, d'une contingence ou d'une genèse historique, ou d'un déterminisme sccial, on s'est attaché à étudier 
le fonctionnement des distributions spatiales et l'évolution des trames pour elles-mêmes. Cela a donné des résultats intéressants en termes de stabilité de ces structures ou trames spatiales au cours du temps d'une part, en termes d'invariance de ces trames lorsqu'on passe d'une portion de la surface terrestre à une autre d'autre part.

La stabilité temporelle suggère la pertinence du concept d'un système spatial qui se maintient dans sa forme et s'auto-organise, fécondité qui ne relève pas sans doute de l'explication au sens de l'interprétation ultime acceptable par tous, mais de l'efficacité opératoire, au sens où simuler c'est déjà en partie comprendre.

L'invariance d'un pays à l'autre, d'un système de production à l'autre assure bien, s'il en était besoin encore, la pertinence et l'utilité d'une analyse des systèmes spatiaux, en partie distincte de l'analyse sociale.

\section{Références.}

Allen P.,1981: "Self-organization in Complex Sytems". Communication au colloque Thermodynamique et sciences de l'homme, Université de Créteil, 22-23 juin, 12 p.

Beguin H., 1979: "Urban Hierarchy and the Rank-size Distribution". Geographical Analysis, Vol. $11, n^{0} 2$, pp. 149-163.

Berry B. J. L., 1967: Geographie des marchés et du commerce de détail. Traduction française, Paris, A. Colin, 1972.

Berry B. J. L., 1972: City Classification Hand-book: Methods and Applications. New York, Wiley, $380 \mathrm{p}$.

Berry B. J. L. et Horton F. E., 1970: Geographic Perspectives on Urban Systems. Prentice Hall, $564 \mathrm{p}$.

Bose N. K., 1968: Calcutta 1964: a Social Survey. Calcutta, Lalvani Publishing House.

Brunet R., 1967: Les phénomènes de discontinuité en géographie. Paris, C.N.R.S., Mémoires et documents, volume $7,117 \mathrm{p}$.

Bussière R. et Stovall T., 1981: Systèmes évolutifs urbains et régionaux à l'état d'équilibre. Paris, C.R.U., 298 p.

Cauvin C., 1984: La perception des distances en milieu intra-urbain. Paris, C.N.R.S., Mémoires et documents, à paraître.

Cauvin C. et Reymond H., 1980: "Cartographie informatisée en géographie humaine". C.N.R.S., A.T.P., Expérimentations en cartographie transformationnelle, t.2, 150 p.

Grafmeyer Y. J., 1979: L'école de Chicago, naissance de l'écologie urbaine. Paris, Editions du Champ urbain.

Guermond Y., 1981: Micro-informatique et analyse multivariée: la structure socio-professionnelle de l'agglomération de Rouen. Brouillons Dupont.

Hautreux J., Lecourt R. et Rochefort M., 1963: Le niveau supérieur de l'armature urbaine française. Paris, Ministère de la construction, Commission de l'équipement urbain, $60 p$. 
Henry L. et Guttierez H., 1977: "Qualité des prévisions démographiques à court terme". Population, no3, pp. 625-647.

Klein Ch. et Pruvot M., 1984: "De l'invariance en écologie urbaine factorielle", L'Espace géographique, à parấtre.

Noin D. et Chauviré Y., 1980: "La structure socio-professionnelle de l'agglomération parisienne". Bulletin de l'Association des Géographes français.

Pumain D., 1982: La dynamique des villes. Paris, Economica, $231 \mathrm{p}$.

Pumain D., 1984: "L'évolution séculaire de la trame urbaine", in Théo Quant, Géoscopie de la France, Paris, Minard.

Pumain D. et Saint-Julien Th., 1978: Les dimensions du changement urbain, Paris, C.N.R.S., 202 p.

Racine J.-B., 1973: Un type nord-américain d'expansion métropolitaine: la commune urbaine du grand Montréal, Lille 1975, Atelier de reproduction des thèses.

Racine J.-B., 1973: "La centralité comerciale relative des municipalités du système métropolitain montréalais". L'Espace géographique, $n^{\circ} 4$, pp. 275-289.

Robic M.-C., 1982: "Cent ans avant Christaller... Une théorie des lieux centraux". L'Espace géographique, $n^{0} 1, p p .5-12$.

Saint-Julien Th., 1982: Croissance industrielle et système urbain, Paris, Econonica, 280 p.

Skinner G. W., 1984: "Marketing and Social Structure in Rural China". Journal of Asian Studies, vol. 34 .

Vance J. E., 1962: "Emerging Patterns of Commercial Structure in American Cities". Land Studies in Geography, série $B, n^{\circ} 24$.

Vigouroux M. et Volle J.P., 1982: "L'espace social des centres et quartiers traditionnels à Nímes, Montpellier et Perpignan: un essai d'écologie factorielle". Bulletin de la société languedocienne de géographie, pp. 287-320. 